\title{
Route Redistribution-A Case Study
}

\author{
Vishesh $S^{1}$, Chethan M Yadav ${ }^{2}$, Nagaraj Manjunath Moger $^{2}$, Chiranjeevi $S^{2}$, Akshay Bhat ${ }^{2}$, Gagan $\mathbf{M}^{2}$ \\ B.E, Department of Telecommunication Engineering, BNM Institute of Technology, Bangalore, India ${ }^{1}$ \\ Student, Department of Electronics and Communication Engineering, BMSCE, Bangalore, India ${ }^{2}$
}

\begin{abstract}
Network layer is the third layer in the OSI model [1]. The network layer provides connectivity and path selection between two host systems. It is responsible for routing data packets and selecting the best path to deliver data. The network layer prioritizes data known as Quality of Service (QOS) [2]. Routers [3] and Layer 3 switches operate at the network layer. A Router can also act as a Layer 2 and Layer 1 device. A routing protocol [4] specifies how routers communicate with each other. A routing protocol is a set of rules used by routers to determine the most appropriate path into which they should forward packets towards their intended destination. In this paper we give you a brief introduction on redistribution. Using a routing protocol to advertise routes that are learned by some other means, such as by another routing protocol, static routes or directly connected routes, is called redistribution. This situation never arises in the case of single protocol routing environment but multi-protocol routing is common for a number of reasons like company mergers, multiple departments managed by multiple network administrators, and multi-vendor environments. Route redistribution allows different routing protocols to exchange routing information. In this paper we have considered one such situation of route redistribution. Redistribution is done at the boundary routers at the boundary of two merged companies- company A and company B, adapting OSPF and EIGRP routing protocols respectively in their respective AS.
\end{abstract}

Keywords: Network layer, OSI model, Quality of Service (QOS), Routers and Layer 3 switches, multi-protocol routing, redistribution, OSPF and EIGRP.

\section{INTRODUCTION}

The OSI model consists of 7 layers. A layered network model is used to reduce complexity, standardize interfaces, to facilitate modular engineering, simplify learning and working.

Following are the seven layers of the OSI model

- Layer 1- Physical Layer- It is responsible for binary transmission. Defines the electrical, mechanical, procedural and functional specifications for activating, maintaining and deactivating the physical link.

- Layer 2- Data link Layer- It defines how data is formatted for transmission and how an access to the network is controlled.

- Layer 3- Network Layer- It provides connectivity and path selection between two host systems, routes data packets, best path selection to deliver data and prioritizes data known as QOS.

- Layer 4- Transport Layer- It handles transportation issues between hosts, ensures data transport reliability, establishes, maintains and terminates virtual circuits, information flow control and provides reliability through fault detection and recovery.

- Layer 5, 6 and 7- Session Layer, Presentation Layer and Application Layer respectively.

A router is an electronic device and/or software that connects at least two networks and forwards packets among them according to the information in the packet headers and the routing table. There are many routing protocols to/which specify how routers communicate with each other. A routing protocol is a set of rules used by routers to determine the most appropriate path to which packet is to be forwarded from source to the destination. Following are the routing protocols

- RIP version 1 and 2 [5]

- EIGRP [6]

- OSPF [7]

- IS-IS [8]

- BGP [9]

RIP, EIGRP, OSPF and IS-IS are Interior Gateway Protocols and BGP is an Exterior Gateway Protocol. IGPs are used inside an Autonomous Systems and BGP is used for communication between different Autonomous Systems (AS). 


\section{IP ADDRESSING AND IANA}

The Internet Assigned Numbers Authority (IANA) is a non-profit corporation that is involved in the following activities [10].

\section{- $\quad$ Domain Names}

Management of DNS Root, the .int and .erpa domains

The DNS Root zone

The root is the upper-most part of the DNS hierarchy, and involves delegating administrative responsibility of "toplevel domains", which are the last segment of a domain name, such as .com, .uk and .nz. Part of their tasks includes evaluating requests to change the operators of country code domains, as well as day-to-day maintenance of the details of the existing operator

\section{.INT}

The .int top-level domain, designed for the sole use of cross-national organizations, such as treaty organizations, that do not naturally fit into a specific country's top-level domain. For example, who.int for the World Health Organization.

\section{.ARPA}

The .arpa domain is used internally by Internet protocols, such as for reverse mapping of IP address and delivery of ENUM phone number mapping. We administer this domain in close liaison with the Internet Architecture Board, which has policy responsibility for .arpa.

IDN Practices Repository

To help foster the development of Internationalized Domain Names (IDNs), repository of "IDN tables" which document the permissible characters for different languages and scripts provided for registration by different top-level domain registries. The repository is informative, and designed for information sharing.

Root Key Signing key

The root key signing key provides verification of the DNSSEC-signed root zone.

Special Purpose Domains

A number of special domains are reserved or managed for demonstration purposes, or for future use.

- Number Resources

Co-ordination of the global pool of IP and AS numbers, primarily providing them to Regional Internet Registers.

- $\quad$ Protocol Assignments

Internet protocol's numbering systems are managed in conjunction with standards bodies.

- $\quad$ Classful and Classless IP addresses

An IP address is a 32-bit address that identifies a connection to the internet.

Table 1 shows the IP address classes and Table 2 shows private IP addresses.

Table 1

\begin{tabular}{|c|c|c|c|c|}
\hline Class & $\begin{array}{l}\mathbf{1}^{\text {st }} \text { Octet Decimal } \\
\text { Range }\end{array}$ & $\begin{array}{l}1^{\text {st }} \text { Octet High Order } \\
\text { Bits }\end{array}$ & $\begin{array}{l}\text { Network/Host ID } \\
\text { (N=Network, H=Host) }\end{array}$ & Default Subnet Mask \\
\hline $\mathrm{A}$ & $1-126^{*}$ & 0 & N.H.H.H & 255.0 .0 .0 \\
\hline $\mathrm{B}$ & $128-191$ & 10 & N.N.H.H & 255.255 .0 .0 \\
\hline $\mathrm{C}$ & $192-223$ & 110 & N.N.N.H & 255.255 .255 .0 \\
\hline $\mathrm{D}$ & $224-239$ & 1110 & \multicolumn{2}{|c|}{ Reserved for multicasting } \\
\hline $\mathrm{E}$ & $240-254$ & 1111 & \multicolumn{2}{|c|}{ Used for research } \\
\hline
\end{tabular}

Table 2

\begin{tabular}{|l|l|l|}
\hline Class & Private Networks & Subnet Masks \\
\hline A & 10.0 .0 .0 & 255.0 .0 .0 \\
\hline B & $172.16 .0 .0-172.31 .0 .0$ & 255.24 .0 .0 \\
\hline C & 192.168 .0 .0 & 255.255 .0 .0 \\
\hline
\end{tabular}

\section{III.REDISTRIBUTION}

Consider a company A whose routers are configured with OSPF routing protocol and enclosed within area 0 . Consider another company B using EIGRP as the Internal routing protocol. OSPF and EIGRP are configured within the AS of company A and company B respectively. Due to some reason company A decides to merge with company B. In this 
case we have to connect networks running different routing protocols. Route redistribution allows different routing protocols to exchange routing information. Each routing protocol on a network is separated into an Autonomous System (AS). All routers in the same autonomous system have complete knowledge of the entire AS. Figure 1 shows the network topology of the merger between company A and company B. Route redistribution needs to be carried out at the boundary routers R5 and R6.

The general procedure followed is

- Locate the boundary router(s). This is the router where redistribution will be configured.

- Choose a protocol to be the core (backbone protocol). A general rule of thumb is to pick OSPF or EIGRP.

- Determine the edge protocol. If you are migrating, this is the protocol that will be phased out.

- Configure the router to redistribute from the edge protocol to the backbone protocol.

- Configure the router to redistribute from the backbone protocol to the edge protocol.

- Check redistribution by using the show route command.

\section{IV.RESULTS AND FINDINGS}

Figure 2, 3 and 4 shows the redistribution being done on boundary router R5. Similarly redistribution is carried out on router R6 which is the other boundary router between company A and company B.

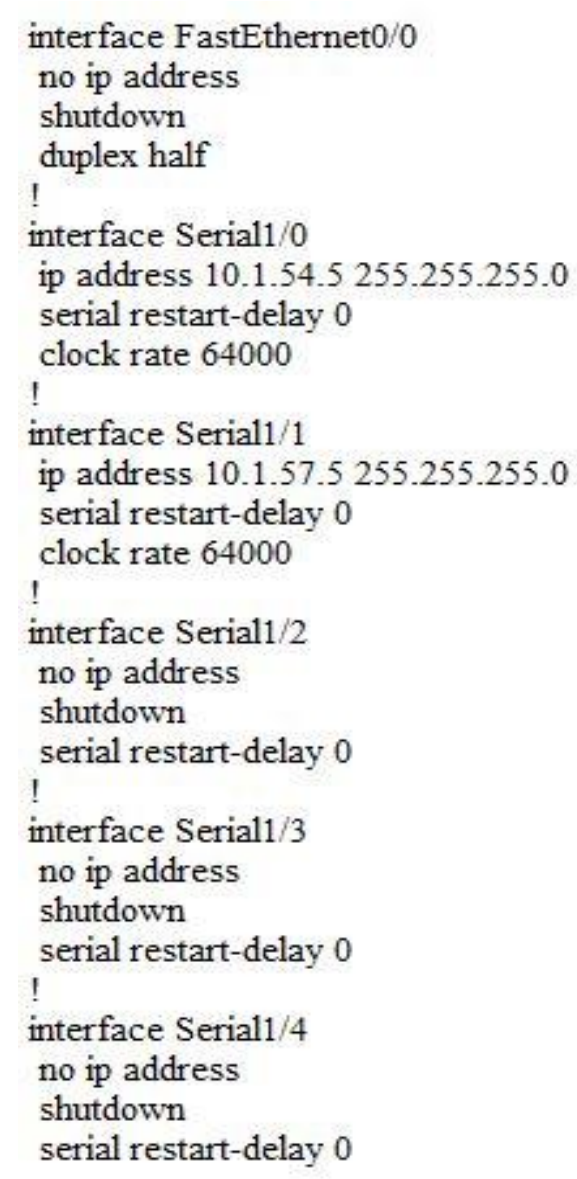

Figure 2 shows the redistribution being done on boundary router R5

\section{CONCLUSIONS}

In this paper a typical example of route redistribution at the boundary router(s) was considered and configured. An example of a company A merging with company B where, company A uses OSPF as its IGP within its AS and company B uses EIGRP as its IGP within its AS was taken. After the company merger redistribution had to be done for successful communication between the routers belonging to two different AS and following two different routing protocols. 


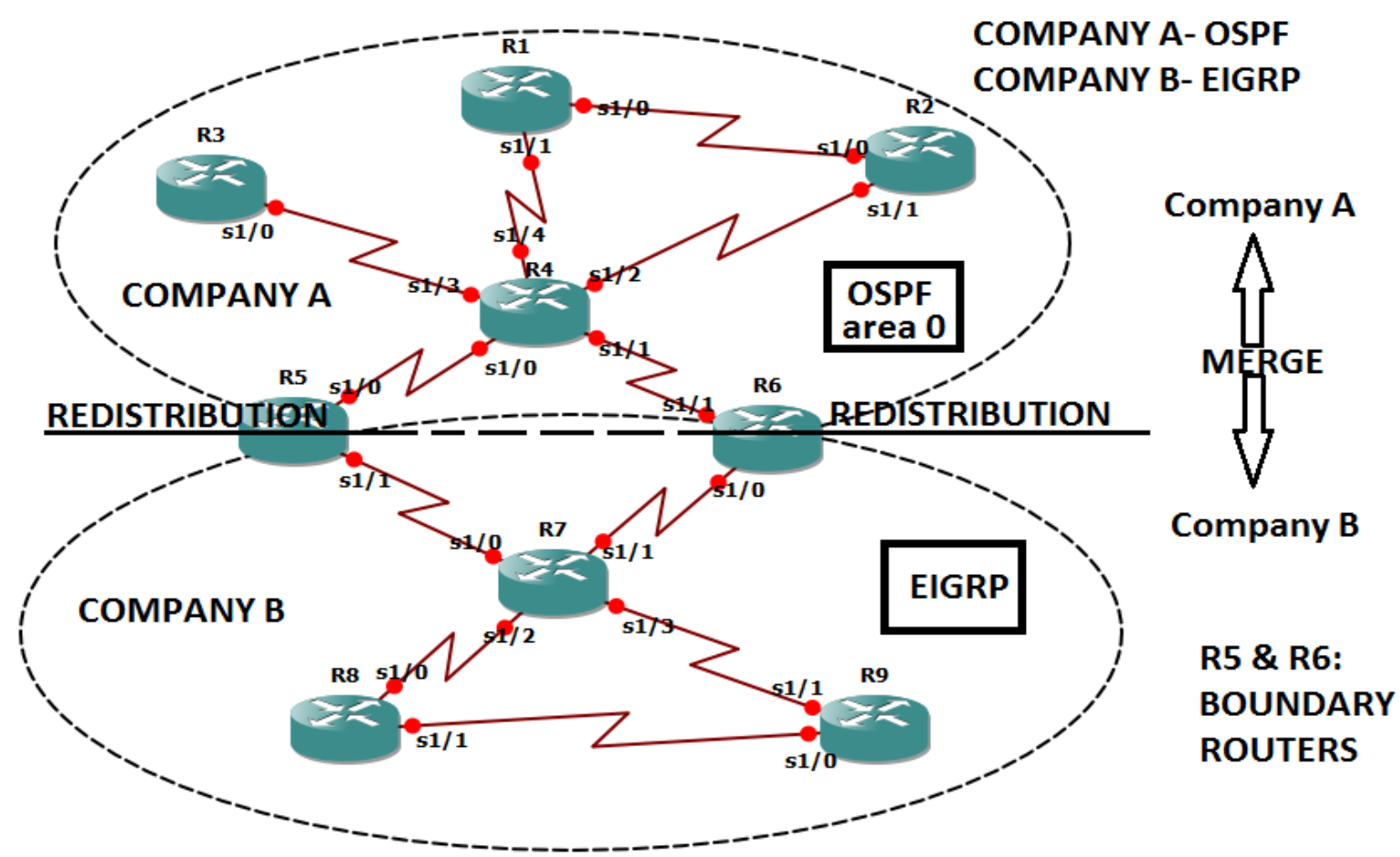

Figure 1 shows the network topology of the merger between Company A and Company B

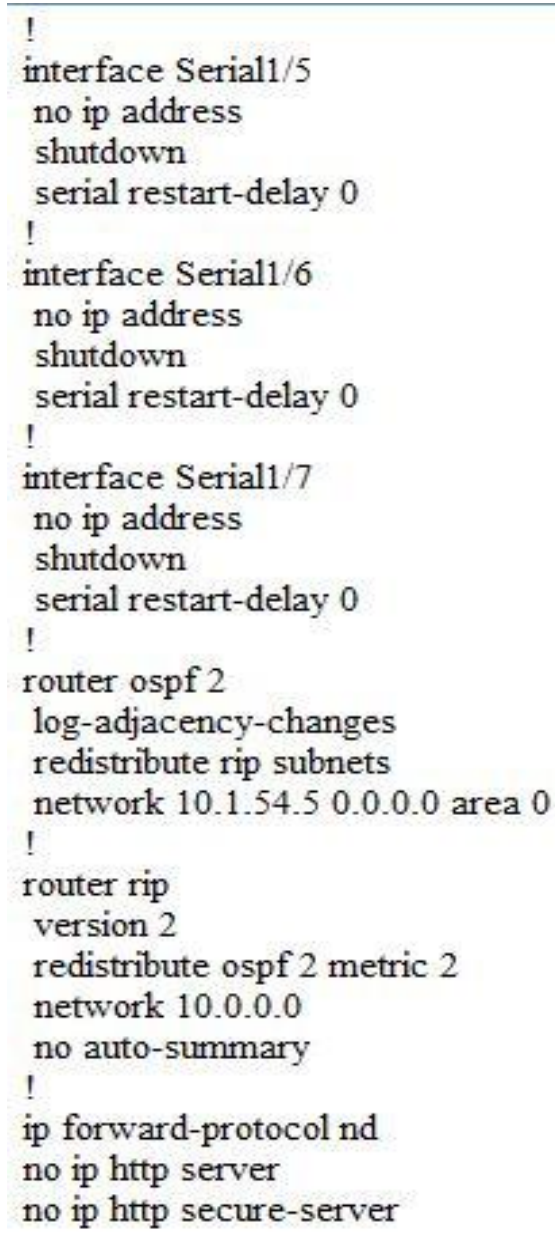

Figure 3 shows the redistribution being done on boundary router R5 (cont.) 


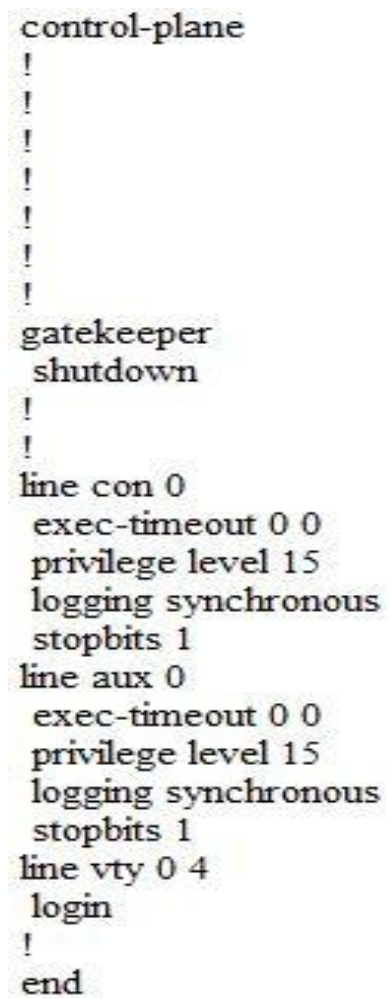

Figure 4 shows the redistribution being done on boundary router R5 (cont.)

\section{REFERENCES}

[1] Internetworking Basics - Cisco- https://www.cisco.com/cpress/cc/td/cpress/fund/ith/ith01 gb.html

[2] Quality of Service (QoS) - Cisco- www.cisco.com > ... > Cisco IOS and NX-OS Software > Cisco IOS Technologies

[3] Routers - Cisco- www.cisco.com/c/en_in/products/routers/index.html

[4] Cisco - Routing Protocols- www.cisco.com/public/technotes/tech_protocol.shtml

[5] Difference between RIPv1 and RIPv2 - Basic Cisco CCNA CCNP ...- basic-ccna-ccnp-commands.blogspot.com/p/difference-between-ripv1-andripv2.html

[6] Survey on the RIP, OSPF, EIGRP Routing Protocols - ijcsit- ijcsit.com/docs/Volume\%205/vol5issue02/ijcsit2014050222.pdf

[7] Open Shortest Path First (OSPF) - Cisco- www.cisco.com > ... > Cisco IOS Technologies > IP Routing and Services > IP Routing

[8] OSI IS-IS intra-domain routing protocol

D Oran - 1990 - tools.ietf.org

[9] Border Gateway Protocol (BGP): A Boon to Internet Application and ISPs-Vishesh S1, Manu Srinath1, Suhas S2, Srivatsa S Murthy2, Amar Tejas M3- DOI 10.17148/IJARCCE.2016.51109

[10] IANA- https://www.iana.org/about

\section{BIOGRAPHIES}

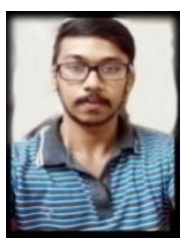

VISHESH S born on $13^{\text {th }}$ June 1992, hails from Bangalore (Karnataka) and has completed B.E in Telecommunication Engineering from VTU, Belgaum, Karnataka in 2015. He also worked as an intern under Dr Shivananju BN, Department of Instrumentation, IISc, Bangalore. His research interests include Embedded Systems, Wireless Communication and Medical Electronics. He is also the Founder and Managing Director of the company Konigtronics Private Limited. He has guided over a hundred students/lecturers/interns/professionals in their research works and projects. He is also the co-author of many International Research Papers. He is currently pursuing MBA. Presently Konigtronics Private Limited has extended its services in the field of Real Estate, Webpage Designing and Entrepreneurship.

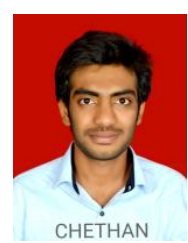

CHETHAN M YADAV hails from Bengaluru, Karnataka. He is currently pursuing BE in Electronics and Communication Engineering at BMSCE, Bengaluru. His areas of interest include embedded systems, networking. 


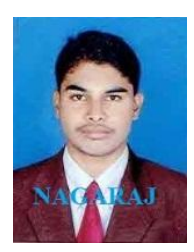

NAGARAJ MANJUNATH MOGER hails from Bengaluru, Karnataka. He is currently pursuing BE in Electronics and Communication Engineering at BMSCE, Bengaluru. His areas of interest include embedded systems, networking.

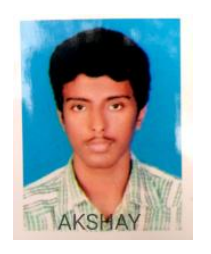

AKSHAY BHAT hails from Bengaluru, Karnataka. He is currently pursuing BE in Electronics and Communication Engineering at BMSCE, Bengaluru. His areas of interest include embedded systems and networking.

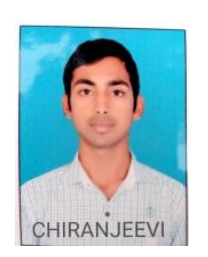

CHIRANJEEVI S hails from Bengaluru, Karnataka. He is currently pursuing BE in Electronics and Communication Engineering at BMSCE, Bengaluru. His areas of interest include control systems, embedded systems and networking.

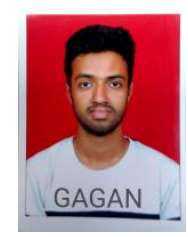

GAGAN M hails from Bengaluru, Karnataka. He is currently pursuing BE in Electronics and Communication Engineering at BMSCE, Bengaluru. His areas of interest include embedded systems and networking. 